\title{
The c ring of the F1Fo ATP synthase forms the mitochondrial permeability transition pore: a critical appraisal
}

\author{
Andrew P. Halestrap* \\ School of Biochemistry and Bristol CardioVascular, University of Bristol, Bristol, UK \\ ${ }^{*}$ Correspondence: a.halestrap@bristol.ac.uk \\ Edited and reviewed by: \\ Paolo Pinton, University of Ferrara, Italy
}

Keywords: mitochondrial permeability transition pore, necrotic cell death, F1Fo-ATPase, proteoliposomes, commentary

\section{A commentary on}

An uncoupling channel within the csubunit ring of the F1Fo ATP synthase is the mitochondrial permeability transition pore

by Alavian KN, Beutner G, Lazrove E, Sacchetti S, Park HA, Licznerski P, Li H, Nabili P, Hockensmith K, Graham M, Porter GA Jr., Jonasa EA. Proc Natl Acad Sci U S A (2014) 111:10580-5. doi:10.1073/ pnas. 1401591111

The mitochondrial permeability transition pore (MPTP) is a non-specific pore in the inner mitochondrial membrane (IMM) whose opening is triggered by high matrix $\left[\mathrm{Ca}^{2+}\right]$ to which it is sensitized by [Pi] and oxidative stress. MPTP opening plays a critical role in necrotic cell death such as in cardiac ischemia/reperfusion (I/R) injury and the action of cytotoxic drugs. Indeed MPTP inhibition with cyclosporine A (CsA) protects tissues from $I / R$ injury (1). Matrix cyclophilin D (CyP-D), the target of CsA, facilitates MPTP opening but the identity of the pore-forming proteins (to which CyP-D binds) remains unresolved (2-4). Extensive evidence supports the adenine nucleotide translocase (ANT) being the site of inhibition by adenine nucleotides and bongkrekic acid (BKA - an ANT ligand) and activation by carboxyatractyloside (CAT - another ANT ligand) and oxidative stress (5). Furthermore, the ANT binds CyP-D and, when reconstituted into proteoliposomes, it produces $\mathrm{Ca}^{2+}$. activated pores similar to the MPTP (6, 7). In addition, liver mitochondria lacking ANT1 and ANT2 exhibit MPTP opening that is insensitive to adenine nucleotides, CAT and BKA, and requires higher $\left[\mathrm{Ca}^{2+}\right]$ than control mitochondria (8). However, since pore opening can still be observed, other IMM proteins must be able to form the MPTP. One candidate is the mitochondrial phosphate carrier ( $\mathrm{PiC}$ ), which binds CyP-D, more so following oxidative stress, and may be the locus of MPTP activation by $\mathrm{Pi}(9,10)$. However, neither partial knockdown nor over-expression of $\mathrm{PiC}$ in cell lines affected MPTP opening $(10,11)$, although MPTP opening in heart mitochondria from mice with cardiac-specific $\mathrm{PiC}$ knockout were less calcium sensitive (12). Overall, the available data suggest that the ANT and PiC play roles in MPTP opening but that another IMM protein must also be involved. Several recent papers suggest that this may be the F1Fo ATP synthase.

CyP-D was shown to bind to the F1Fo ATP synthase and modulate its hydrolytic activity $(13,14)$ and in 2013 , the laboratories of both Bernardi (15) and Pinton (16) presented data that implicated the ATP synthase in MPTP formation. Bernardi and colleagues (15) detected $\mathrm{Ca}^{2+}$-activated channels, similar to the MPTP, in phospholipid bilayers containing reconstituted dimers of mammalian F1Fo ATP synthase. Similar channel activity was demonstrated in yeast mitochondria and this was strongly attenuated in mutants lacking the $\varepsilon$ and $\gamma$ subunits needed for ATP synthase dimer formation (17). However, high levels $(0.3 \mathrm{mM})$ of $\mathrm{Ca}^{2+}$ were required for channel opening which, unlike MPTP opening, also required $\mathrm{Bz}-423$. No data were presented on the effects of oxidative stress, CsA, or recombinant CyP-D. Furthermore, Pinton and colleagues (18) pointed out that Bernardi's laboratory had previously demonstrated
MPTP opening in $\rho^{0}$ cells, which lack the mitochondrial DNA encoding the $\alpha$ and A6L subunits of the ATP synthase. In addition, the ATPase inhibitor protein F1, which promotes ATP synthase dimerization, attenuates rather than promotes MPTP opening, and enhances cell survival under ischemic conditions (19). Rather, Pinton and colleagues (16) implicated the c-subunits of the Fo ATPase in MPTP formation, showing that their knockdown reduced MPTP opening in response to ionomycin or hydrogen peroxide and their over-expression enhanced opening. The c-subunits form a ring structure in the IMM, and so represent an attractive candidate for forming the MPTP, but direct evidence for this was not provided. However, the paper of Alavian et al. (20) claims to do this.

Alavian et al. (20) confirmed the observations of Bonora et al. (16), but they also reconstituted the purified $\mathrm{c}$-subunit into proteoliposomes and demonstrated channel activity. Most channels conducted at $\sim 100$-pS but a few did so at 1.5-2 nS, similar to the MPTP conductance (21). However, the channels were insensitive to $\mathrm{Ca}^{2+}$ and CsA and were only inhibited by much higher concentrations of ATP and ADP than required to inhibit MPTP opening. The authors proposed that other F1Fo ATP synthase components are needed for MPTP regulation, which they investigated using purified monomeric F1Fo ATP synthase reconstituted into proteoliposomes. Some infrequent channel activity was observed, which was increased by addition of CyP$\mathrm{D}$ and further by $100 \mu \mathrm{M} \mathrm{Ca}{ }^{2+}$. These effects were prevented by $5 \mu \mathrm{M}$ CsA, a concentration much higher than the $\mathrm{K}_{\mathrm{i}}$ for CyP-D (2nM). Channels sensitive to 


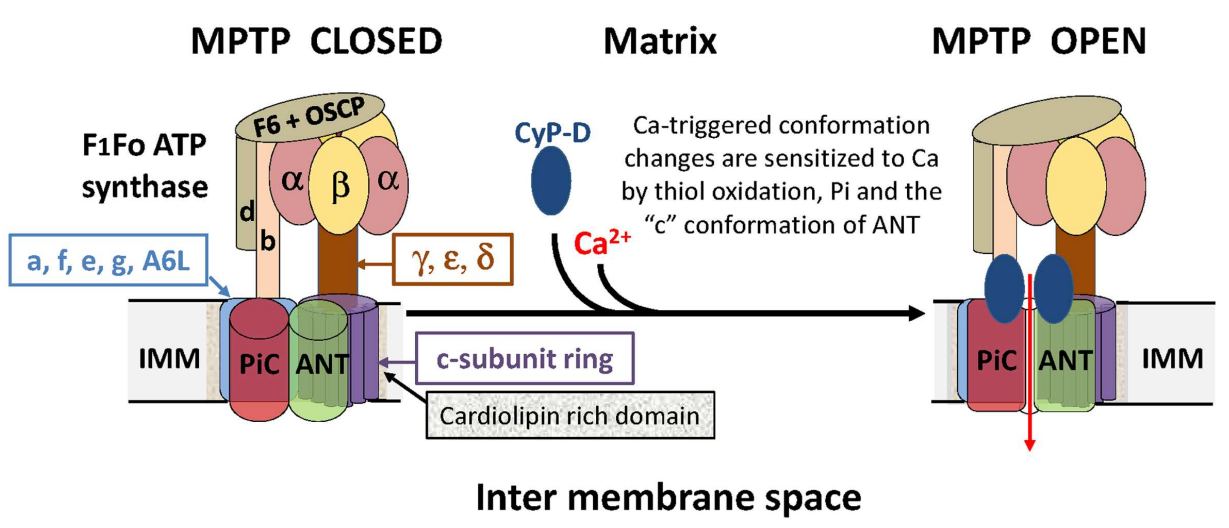

FIGURE 1 | A hypothetical model of the MPTP that proposes an interaction between the ANT, PiC, and F1Fo ATP synthase in the ATP synthasome. It is proposed that the MPTP forms at the interface between interacting domains of the PiC, ANT, and Fo ATP synthase following calcium triggered conformational changes facilitated by the PPlase activity of CyP-D. Note that potential regulatory interactions of the MPTP with outer membrane components are not shown.

both $\mathrm{Ca}^{2+}$ and CsA could also be detected in patches from sub-mitochondrial vesicles (SMVs) enriched in F1Fo ATP synthase or IMMs and these were absent when OSCP and $\beta$ subunits of the ATP synthase and bound CyP-D were removed by urea treatment, while adding back purified $\beta$ subunit to reconstituted c-subunits largely abolished channel activity. However, the authors did not address whether the IMM and SMV preparations also contained ANT and $\mathrm{PiC}$, which is very likely. Rather, they concluded that the sites through which $\mathrm{Ca}^{2+}$, ADP, and CyP-D (and thus CsA) modulate channel activity are on the F1 domain of the ATP synthase whose association with the c-subunit ring may loosen upon $\mathrm{Ca}^{2+}$ and CyP-D binding. This might cause expansion of the ring converting it into a high conductance channel and, using fluorescent probes, they presented evidence consistent with the c-subunits moving apart during MPTP opening. Furthermore, channel activity was greatly enhanced when glycine residues in the c-subunit transmembrane domains were replaced with valines, thus moving the packed helices further apart (20). However, interpretation of these data is difficult because the expressed c-subunits ran at $15 \mathrm{kDa}$ on SDS-PAGE and not $7.6 \mathrm{kDa}$, the size of the mature $\mathrm{c}$ subunit, suggesting that the mitochondrial targeting sequence had not been removed. Indeed several studies in this paper and that of Bonora et al. (16) showed expression of $15 \mathrm{kDa}$ unprocessed protein rather than the true c subunit.
In summary, when the evidence for an involvement of the F1Fo ATP synthase and more specifically its c-subunit in MPTP formation is reviewed critically, it is legitimate to conclude that it is no better than that for the involvement of the ANT and PiC. Perhaps the truth lies in a synthesis, and that an interaction between the ANT, PiC, and F1Fo ATP synthase in the ATP synthasome (22) is critical for MPTP formation, as we (2) and subsequently others $(4,18)$ have concluded. A scheme illustrating how the different components may interact is presented in Figure 1.

\section{REFERENCES}

1. Halestrap AP. A pore way to die: the role of mitochondria in reperfusion injury and cardioprotection. Biochem Soc Trans (2010) 38:841-60. doi:10.1042/BST0380841

2. Halestrap AP. What is the mitochondrial permeability transition pore? J Mol Cell Cardiol (2009) 46:821-31. doi:10.1016/j.yjmcc.2009.02.021

3 . Bernardi P. The mitochondrial permeability transition pore: a mystery solved? Front Physiol (2013) 4:95. doi:10.3389/fphys.2013.00095

4. Karch J, Molkentin JD. Identifying the components of the elusive mitochondrial permeability transition pore. Proc Natl Acad Sci U S A (2014) 111:10396-7. doi:10.1073/pnas.1410104111

5. Halestrap AP, Brenner C. The adenine nucleotide translocase: a central component of the mitochondrial permeability transition pore and key player in cell death. Curr Med Chem (2003) 10:1507-25. doi:10.2174/0929867033457278

6. Brustovetsky $\mathrm{N}$, Becker A, Klingenberg $\mathrm{M}$, Bamberg E. Electrical currents associated with nucleotide transport by the reconstituted mitochondrial ADP/ATP carrier. Proc Natl Acad Sci U S A (1996) 93:664-8. doi:10.1073/pnas.93.2.664
7. Brustovetsky N, Tropschug M, Heimpel S, Heidkamper D, Klingenberg M. A large Ca2+dependent channel formed by recombinant ADP/ATP carrier from Neurospora crassa resembles the mitochondrial permeability transition pore. Biochemistry (2002) 41:11804-11. doi:10. 1021/bi0200110

8. Kokoszka JE, Waymire KG, Levy SE, Sligh JE, Cal JY, Jones DP, et al. The ADP/ATP translocator is not essential for the mitochondrial permeability transition pore. Nature (2004) 427:461-5. doi:10.1038/nature02229

9. Leung AWC, Varanyuwatana P, Halestrap AP. The mitochondrial phosphate carrier interacts with cyclophilin D and may play a key role in the permeability transition. J Biol Chem (2008) 283:26312-23. doi:10.1074/jbc.M805235200

10. Varanyuwatana P, Halestrap AP. The roles of phosphate and the mitochondrial phosphate carrier in the mechanism of the permeability transition. Mitochondrion (2012) 12:120-5. doi:10.1016/ j.mito.2011.04.006

11. Gutierrez-Aguilar M, Douglas DL, Gibson AK, Domeier TL, Molkentin JD, Baines CP. Genetic manipulation of the cardiac mitochondrial phosphate carrier does not affect permeability transition. J Mol Cell Cardiol (2014) 72:316-25. doi:10. 1016/j.yjmcc.2014.04.008

12. Kwong JQ, Davis J, Baines CP, Sargent MA, Karch J, Wang X, et al. Genetic deletion of the mitochondrial phosphate carrier desensitizes the mitochondrial permeability transition pore and causes cardiomyopathy. Cell Death Differ (2014) 21:1209-17. doi:10.1038/cdd.2014.36

13. Giorgio V, Bisetto E, Soriano ME, Dabbeni-Sala F, Basso E, Petronilli V, et al. Cyclophilin D modulates mitochondrial F0F1-ATP synthase by interacting with the lateral stalk of the complex. J Biol Chem (2009) 284:33982-8. doi:10.1074/jbc.M109. 020115

14. Chinopoulos C, Konrad C, Kiss G, Metelkin E, Torocsik B, Zhang SF, et al. Modulation of F0F1-ATP synthase activity by cyclophilin $\mathrm{D}$ regulates matrix adenine nucleotide levels. 
FEBS J (2011) 278:1112-25. doi:10.1111/j.17424658.2011.08026.x

15. Giorgio V, von Stockum S, Antoniel M, Fabbro A, Fogolari F, Forte M, et al. Dimers of mitochondrial ATP synthase form the permeability transition pore. Proc Natl Acad Sci US A (2013) 110:5887-92. doi:10.1073/pnas.1217823110

16. Bonora M, Bononi A, De Marchi E, Giorgi C, Lebiedzinska M, Marchi S, et al. Role of the c subunit of the Fo ATP synthase in mitochondrial permeability transition. Cell Cycle (2013) 12:674-83. doi:10.4161/cc.23599

17. Carraro M, Giorgio V, Sileikyte J, Sartori G, Forte M, Lippe G, et al. Channel formation by yeast F-ATP synthase and the role of dimerization in the mitochondrial permeability transition. J Biol Chem (2014) 289:15980-5. doi:10.1074/jbc.C114. 559633

18. Bonora M, Wieckowski MR, Chinopoulos C, Kepp O, Kroemer G, Galluzzi L, et al. Molecular mechanisms of cell death: central implication of ATP synthase in mitochondrial permeability transition. Oncogene (2014). doi:10.1038/onc.2014.96
19. Campanella M, Casswell E, Chong S, Farah Z, Wieckowski MR, Abramov AY, et al. Regulation of mitochondrial structure and function by the F1FoATPase inhibitor protein, IF1. Cell Metab (2008) 8:13-25. doi:10.1016/j.cmet.2008.06.001

20. Alavian KN, Beutner G, Lazrove E, Sacchetti S, Park HA, Licznerski P, et al. An uncoupling channel within the c-subunit ring of the F1FO ATP synthase is the mitochondrial permeability transition pore. Proc Natl Acad Sci U S A (2014) 111:10580-5. doi:10.1073/pnas.1401591111

21. Zoratti M, De Marchi U, Biasutto L, Szabo I. Electrophysiology clarifies the megariddles of the mitochondrial permeability transition pore. FEBS Lett (2010) 584:1997-2004. doi:10.1016/j.febslet.2010. 01.012

22. Chen C, Ko Y, Delannoy M, Ludtke SJ, Chiu W, Pedersen PL. Mitochondrial ATP synthasome: three-dimensional structure by electron microscopy of the ATP synthase in complex formation with carriers for Pi and ADP/ATP. J Biol Chem (2004) 279:31761-8. doi:10.1074/jbc. M401353200
Conflict of Interest Statement: The author declares that the research was conducted in the absence of any commercial or financial relationships that could be construed as a potential conflict of interest.

Received: 07 August 2014; paper pending published: 07 August 2014; accepted: 11 August 2014; published online: 25 August 2014.

Citation: Halestrap AP (2014) The cring of the F1Fo ATP synthase forms the mitochondrial permeability transition pore: a critical appraisal. Front. Oncol. 4:234. doi: 10.3389/fonc.2014.00234

This article was submitted to Molecular and Cellular Oncology, a section of the journal Frontiers in Oncology. Copyright $\odot 2014$ Halestrap. This is an open-access article distributed under the terms of the Creative Commons Attribution License (CC BY). The use, distribution or reproduction in other forums is permitted, provided the original author(s) or licensor are credited and that the original publication in this journal is cited, in accordance with accepted academic practice. No use, distribution or reproduction is permitted which does not comply with these terms. 